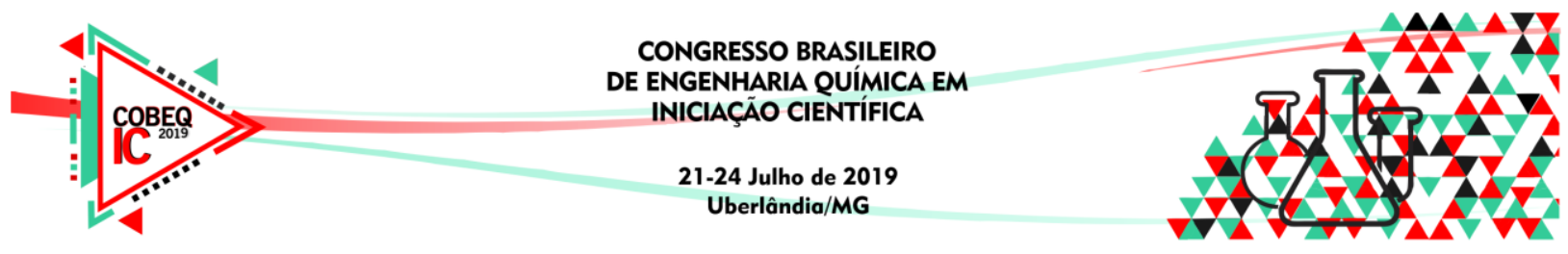

\title{
ANÁlISE DA PRODUÇÃO DE GRAFENO ATRAVÉS DA CARBONIZAÇÃO DA CINZA DE CASCA DO ARROZ
}

\author{
D.M. PEREZ ${ }^{1}$, L.S. SEVERO ${ }^{2}$, D.A. CAMPANELLI ${ }^{3}$ e L.E.G. ARMAS ${ }^{4}$ \\ ${ }^{1}$ Universidade Federal do Pampa, Programa de Pós-Graduação em Engenharia \\ ${ }^{2}$ Universidade Federal do Pampa, Programa de Pós-Graduação em Engenharia \\ ${ }^{3}$ Universidade Federal do Pampa, Faculdade de Engenharia Mecânica \\ ${ }^{4}$ Universidade Federal do Pampa, Programa de Pós-Graduação em Engenharia \\ E-mail para contato: diegoperez.ec@gmail.com
}

\begin{abstract}
RESUMO - A casca de arroz (CA) é um resíduo agrícola oriundo do beneficiamento do arroz que corresponde em média $20 \%$ do peso do grão, sendo estimado para o ano de 2017 a produção de aproximadamente 754,6 milhões de toneladas de arroz. Vários estudos demonstram a possibilidade de obtenção de grafeno através da cinza da casca do arroz (CCA) com baixo custo de produção em comparação com outros métodos já existentes. Este trabalho tem por objetivo realizar um estudo da síntese e caracterização da CCA com alto teor de carbono utilizando diferentes métodos de queima em diferentes temperaturas $\left(400{ }^{\circ} \mathrm{C}\right.$, $500^{\circ} \mathrm{C}, 600{ }^{\circ} \mathrm{C}$ e $\left.700{ }^{\circ} \mathrm{C}\right)$ com tempo de duração de queima variada $(20,40,60$ e 120 min.). Com a finalidade de obter uma cinza com alto teor de carbono para a fabricação de grafeno, as amostras obtidas foram analisadas usando as técnicas de fluorescência de raios X (FRX), espectroscopia Raman e microscópio eletrônico de varredura (MEV). Finalmente, para obter o grafeno, a CCA com alto teor de carbono foi funcionalizada com hidróxido de potássio $(\mathrm{KOH})$. Resultados das análises por FRX mostraram o aumento da porcentagem de dióxido de silício ( $\mathrm{SiO} 2)$, em todas as amostras, a medida que aumenta a temperatura e o tempo de queima. Com base a estes resultados foi observado que as melhores amostras com alta porcentagem de carbono foram queimadas na temperatura de $500^{\circ} \mathrm{C}$ e tempo de 60 minutos. Por outro lado, análises de espectroscopia Raman da CCA antes e após a ativação com $\mathrm{KOH}$ mostraram a presença da banda $2 \mathrm{D}$ em $\sim 2700 \mathrm{~cm}^{-1}$, evidenciando desta maneira, a obtenção de grafeno a partir da CCA.
\end{abstract}

\section{INTRODUÇÃO}

$\mathrm{O}$ arroz é o cereal mais importante do mundo como principal componente da dieta básica da população. Sua importância mundial não se remete apenas ao volume de produção, mas principalmente, por se constituir no principal alimento do homem, ao lado do trigo. Segundo a FAO - Food and Agriculture Organization of the United Nations, à âmbito mundial a produção de arroz para 2018 está estimada em 769,9 milhões de toneladas, com números superiores ao estimado para 2017. Dados do IRG - Instituto Rio Grandense de Arroz, mostram que o estado do Rio Grande do Sul possui a maior contribuição na produção de arroz do país, chegando em um total de 8,2 milhões de toneladas para a safra de 2017/2018, porém, 


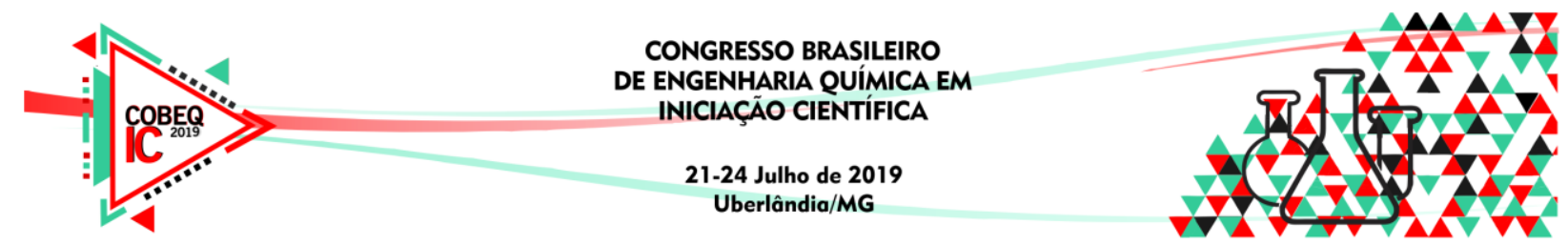

a casca do arroz corresponde a $20 \%$ do peso do grão tornando-se o maior resíduo sólido oriundo do processo de beneficiamento do grão.

Após a queima completa da casca do arroz, cerca de $20 \%$ da casca de arroz é convertida em cinza, o que resulta em um grande volume de resíduo agroindustrial que ainda não possui uma destinação certa, geralmente sendo simplesmente descartada ou lançada em aterros, o que poderá gerar problemas ambientais, tais como poluição de mananciais de água, do ar e do solo. Muramatsu et al. (2014), Sankar et al. (2017), entre outros autores, demonstraram em seus estudos que é possível produzir quantidades em massa de um novo tipo de grafeno com baixo custo de produção, graças ao carbono presente na cinza da casca do arroz, com tratamento químico a base de hidróxido de potássio.

Neste trabalho propõe-se realizar um estudo da síntese e caracterização da cinza da casca de arroz (CCA) com a finalidade da aquisição de grafeno. As amostras produzidas serão analisadas usando as técnicas de fluorescência de raios X (FRX), espectroscopia Raman (RAMAN) e microscópio eletrônico de varredura (MEV). Em sequência, a amostra com alto teor de carbono, obtida no processo de queima, foi ativada (funcionalizada) com hidróxido de potássio $(\mathrm{KOH})$ para a obtenção do grafeno. A comparação das amostras antes e após a ativação com $\mathrm{KOH}$, mostraram a presença da banda 2D, a qual é característica de grafeno.

\section{MATERIAIS E MÉTODOS}

Nesta seção serão apresentados os materiais e métodos utilizados no desenvolvimento deste trabalho, a qual foi dividida em duas etapas, ambas fracionadas em três fases e ilustradas através de um organograma para cada etapa.

\subsection{Materiais}

A casca do arroz utilizada para a pesquisa foi doada pela empresa CERGRAL LTDA, com o plantio localizado na região de Itaqui - RS. O material doado se manteve armazenado em saco de polipropileno, em local seco e arejado, evitando o contato direto com o piso e afastado das paredes. A CA foi usada como matéria prima para a obtenção de carbono amorfo após a carbonização do mesmo.

O hidróxido de potássio $(\mathrm{KOH})$, também chamado de hidróxido cáustico, é uma base de risco corrosivo classe 8 . O produto tem teor de $\geq 85,00 \%$ e se apresenta em forma de lentilhas, foi adquirido da empresa APRO QUÍMICA - Comércio de produtos químicos LTDA, localizada na cidade de Santa Maria - RS, sendo fabricado pela empresa NEON COMERCIAL, localizada em Suzano - SP. Segundo Wang et al (2011), ativação química de várias fontes de carbono usando $\mathrm{KOH}$ como reagente de ativação é muito promissora devido à sua menor temperatura de ativação, maiores rendimentos, distribuição de micro poros bem definida e área superficial ultra alta.

\subsection{Métodos}

A pesquisa foi dividida em duas etapas: Na etapa 1 descreve-se a obtenção da CCA com alto teor de carbono, e na etapa 2 descreve-se a obtenção de grafeno a partir da CCA com a 


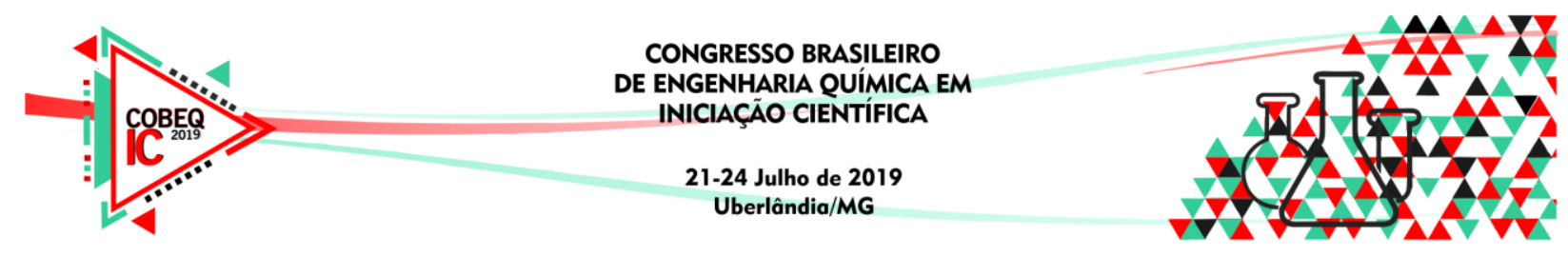

utilização de $\mathrm{KOH}$ como agente de ativação. A CCA selecionada para a obtenção do grafeno foi a que apresentou maior quantidade de carbono e menores porcentagens de sílica em sua composição, tendo em vista o método de queima, temperatura e tempo de duração da queima. A Tabela 1 ilustra a divisão das etapas e fases da pesquisa mencionada anteriormente.

Tabela 1 - Desenvolvimento da Pesquisa

\begin{tabular}{|c|c|c|}
\hline Etapa & Fase & Processo \\
\hline \multirow{3}{*}{ Etapa 1} & 1 & Lavagem e seleção da CA \\
\cline { 2 - 3 } & 2 & Queima da CA em diferentes temperaturas e tempos \\
\cline { 2 - 3 } & 3 & Caracterização da CCA \\
\hline \multirow{3}{*}{ Etapa 2 } & 1 & Ativação da CCA \\
\cline { 2 - 3 } & 2 & Agitação e Filtragem do Grafeno \\
\cline { 2 - 3 } & 3 & Caracterização do Grafeno \\
\hline
\end{tabular}

Etapa 1: A casca foi submetida a três ciclos de lavagem, sendo o primeiro a lavagem com água corrente para remoção de impurezas de maior tamanho e dos ciclos de lavagem com água destilada. Para a síntese da cinza, a casca de arroz limpa foi submetida a três métodos distintos de queima: queima em forno mufla (FN), queima em forno tubular a vácuo (FC) e queima em forno tubular com fluxo contínuo de gás nitrogênio (FG), com a finalidade de determinar o comportamento da casca durante a carbonização em diferentes ambientes. Nos três métodos, as amostras foram queimadas durante 20, 40, 60 e 120 minutos a temperaturas de: $400{ }^{\circ} \mathrm{C}, 500{ }^{\circ} \mathrm{C}, 600{ }^{\circ} \mathrm{C}$ e $700{ }^{\circ} \mathrm{C}$, totalizando 48 amostras de cinzas. Posteriormente, estas amostras foram caracterizadas por FRX, RAMAN e MEV.

Etapa 2: A cinza da casca de arroz com maiores porcentagens de carbono foi misturada com $\mathrm{KOH}$ em uma relação de 1:4 (CCA: $\mathrm{KOH})$ em peso colocados em recipiente de alumina envolto em lã cerâmica dentro do forno mufla (FN) a uma temperatura de $850{ }^{\circ} \mathrm{C}$ por 2 horas. Logo após o resfriamento a amostra foi colocada agitada magnética em $100 \mathrm{ml}$ de água destilada por 6 horas e filtrada para remoção do líquido existente, para depois ser caracterizada por FRX, RAMAN e MEV. As etapas do processo químico para a síntese do material no recozimento em temperatura de $850^{\circ} \mathrm{C}$ e processo de filtragem, como sugere Sankar et al (2017), é mostrado a seguir:

$$
\begin{aligned}
& 6 \mathrm{KOH}+2 \mathrm{C}(\mathrm{Cinza}) \underset{850^{\circ} \mathrm{c}}{\longrightarrow} 2 \mathrm{~K}+3 \mathrm{H}_{2}+2 \mathrm{~K}_{2} \mathrm{CO}_{3} \\
& \mathrm{~K}_{2} \mathrm{CO}_{3}+\mathrm{SiO}_{2} \underset{\mathrm{R} \mathrm{Rn}_{\mathrm{r}}}{\longrightarrow} \mathrm{C}+\mathrm{K}_{2} \mathrm{SiO}_{3}+\mathrm{O}_{2} \\
& \mathrm{~K}_{2} \mathrm{SiO}_{3}+\mathrm{C}+\mathrm{H}_{2} \mathrm{O} \underset{\tau \mathrm{A}}{\longrightarrow} \mathrm{C}+\left(\mathrm{K}_{2} \mathrm{SiO}_{3}+\mathrm{H}_{2} \mathrm{O}\right) \text { solução }
\end{aligned}
$$

\section{RESULTADOS E DISCUSSÕES}

\subsection{Perda de massa no processo de carbonização da CA}

Para o estudo da perda de massa da casca de arroz no processo de queima, as amostras submetidas aos diferentes métodos de queima, temperatura e tempo de permanência foram pesadas antes e após a carbonização. O método de queima utilizando o forno mufla (FN - 


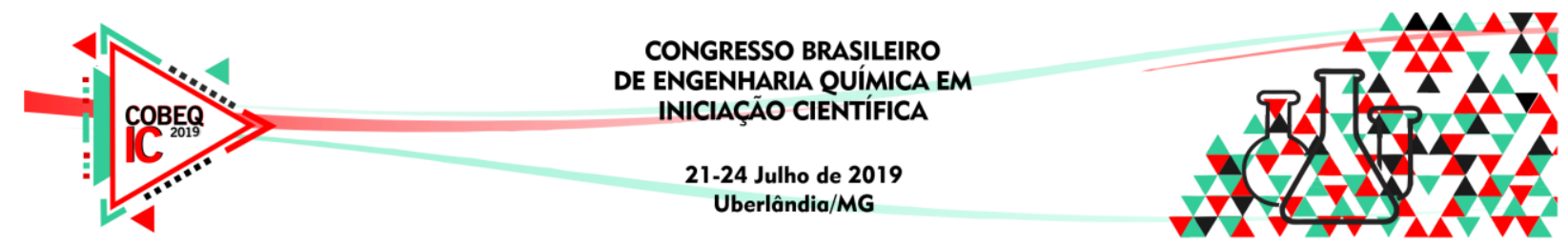

linha vermelha), apresentou piores resultados em relação à perda de massa, queimando mais material em todos os ensaios. Sendo justificado pois, ao contrário dos demais métodos, a queima no forno mufla é feita com a presença de ar no interior do forno que, por sua vez, contém oxigênio fazendo com que haja maior oxidação da casca tendo, como consequência, o consumo de maiores quantidades de matéria orgânica no processo se comparado aos demais métodos onde a presença do oxigênio é removida. A Figura 1 ilustra a porcentagem de material restante pós carbonização juntamente com a coloração característica da casca queimada em diferentes temperaturas.

Figura 1 - Comparação da porcentagem de material restante após carbonização nos três métodos de queima: FN (linha vermelha), FC (linha verde), FG (linha azul)

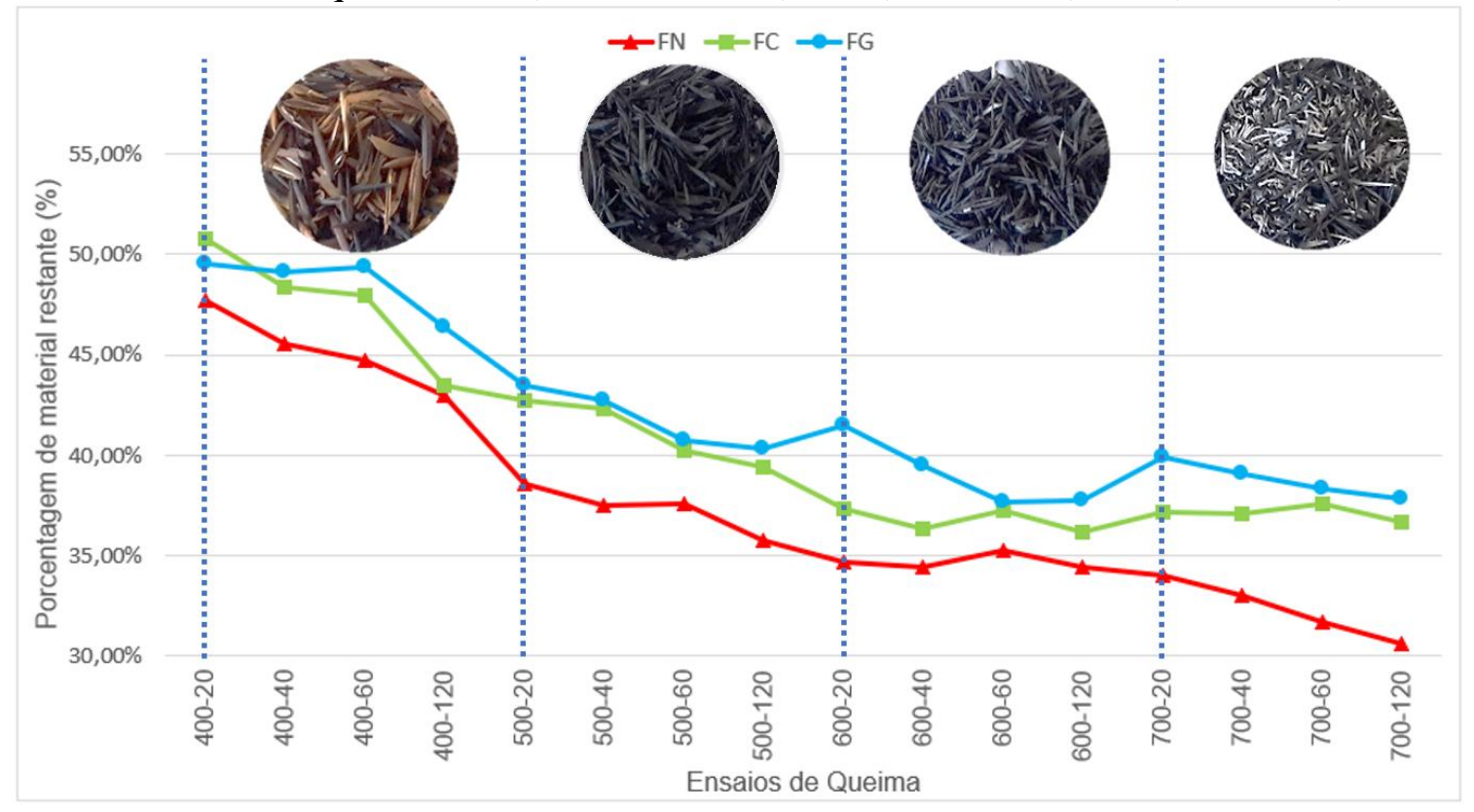

\subsection{Fluorescência de raio $X(F R X)$}

As maiores composições químicas presentes nas amostras de cinza da casca do arroz, entre as oito detectadas, foram: óxido de silício $\left(\mathrm{SiO}_{2}\right)$, óxido de alumínio $\left(\mathrm{Al}_{2} \mathrm{O}_{3}\right)$ e óxido de magnésio $(\mathrm{MnO})$, respectivamente, para os três métodos de queima utilizados, Azizi e Yousefpour (2010) entre outros autores relatam a presença desses mesmos compostos em seus trabalhos, porém com porcentagens diferentes, devido a localização e variedade da matéria prima.

A composição (\% em massa) da casca de arroz, juntamente com as amostras de cinza de casca de arroz com maior conteúdo de carbono estimado para os diferentes métodos de queima foram: em forno tubular com fluxo contínuo de gás nitrogênio à $500{ }^{\circ} \mathrm{C}$ por 120 minutos (FG500-120), em forno tubular a vácuo à $500{ }^{\circ} \mathrm{C}$ por 60 minutos (FC500-60) e em forno mufla à $500^{\circ} \mathrm{C}$ por 20 minutos (FN500-20), respectivamente. Para a obtenção do grafeno (GFN-850) foi utilizado a amostra FC 500-60, pois foi considerada a melhor opção para o processo de fabricação do nano material devido a relação custo/benefício do processo de fabricação, tempo de queima e perda de massa. A porcentagem das composições químicas contidas nas amostras de casca, cinza e grafeno podem ser observadas na Tabela 2. 


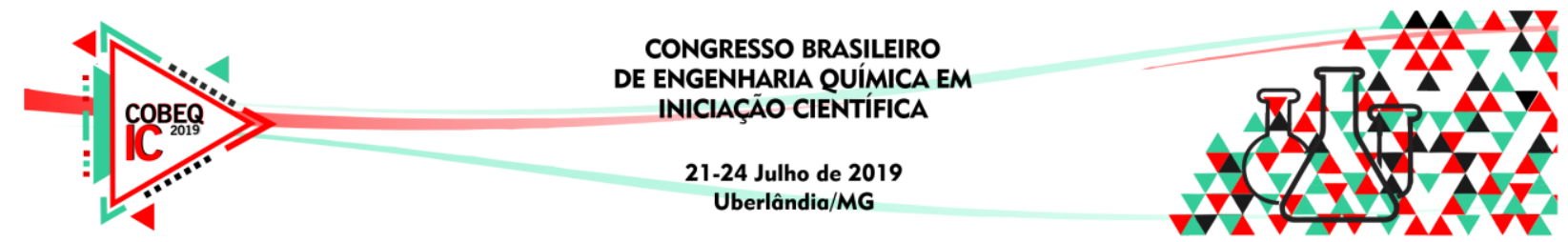

Tabela 2 - FRX das amostras de casca (CA), cinza (FG, FC, FN) e grafeno (GFN)

\begin{tabular}{|c|c|c|c|c|c|c|}
\hline \multirow{2}{*}{ Nome } & \multirow{2}{*}{$\begin{array}{c}\text { Componente } \\
(\% \text { em massa })\end{array}$} & \multicolumn{5}{|c|}{ Ensaios } \\
\cline { 5 - 7 } & & $\mathrm{CA}$ & FG500-120 & FC500-60 & FN500-20 & GFN-850 \\
\hline Óxido de Magnésio & $\mathrm{MgO}$ & 1,23 & 1,85 & 2,01 & 0,87 & 1,09 \\
\hline Óxido de Alumínio & $\mathrm{Al}_{2} \mathrm{O}_{3}$ & 3,45 & 3,45 & 0,62 & 0,66 & 16,50 \\
\hline Óxido de Silício & $\mathrm{SiO}_{2}$ & 26,5 & 46,1 & 49,8 & 54,1 & 12,2 \\
\hline Óxido de Fósforo & $\mathrm{P}_{2} \mathrm{O}_{5}$ & 0,07 & 0,14 & 0,14 & 0,16 & 0,00 \\
\hline Óxido de Potássio & $\mathrm{K}_{2} \mathrm{O}$ & 0,58 & 1,05 & 0,76 & 1,05 & 7,61 \\
\hline Óxido de Cálcio & $\mathrm{CaO}$ & 0,35 & 0,74 & 0,83 & 0,82 & 0,69 \\
\hline Óxido de Manganês & $\mathrm{MnO}^{n}$ & 0,09 & 0,24 & 0,23 & 0,3 & 0,29 \\
\hline Óxido de Ferro & $\mathrm{Fe}_{2} \mathrm{O}_{2}$ & 1,33 & 0,03 & 0,05 & 0,03 & 0,06 \\
\hline
\end{tabular}

\subsection{Microscópio Eletrônico de Varredura (MEV)}

O microscópio eletrônico de varredura possibilita a observação de estruturas tridimensionais com elevado índice de detalhamento da amostra, baseando-se na interação de elétrons incidentes sobre a matéria. A Figura 2 ilustra a cinza da casca do arroz, com magnitude de 2000x antes da ativação (a) e após ativação com $\mathrm{KOH}$ (b), onde pode ser observado, no círculo vermelho, a formação de vários poros ao longo de toda a superfície da amostra como relatado por Wang et al. (2011).

Figura 2 - Imagem MEV: (a) CCA antes da ativação; (b) CCA após ativação com KOH
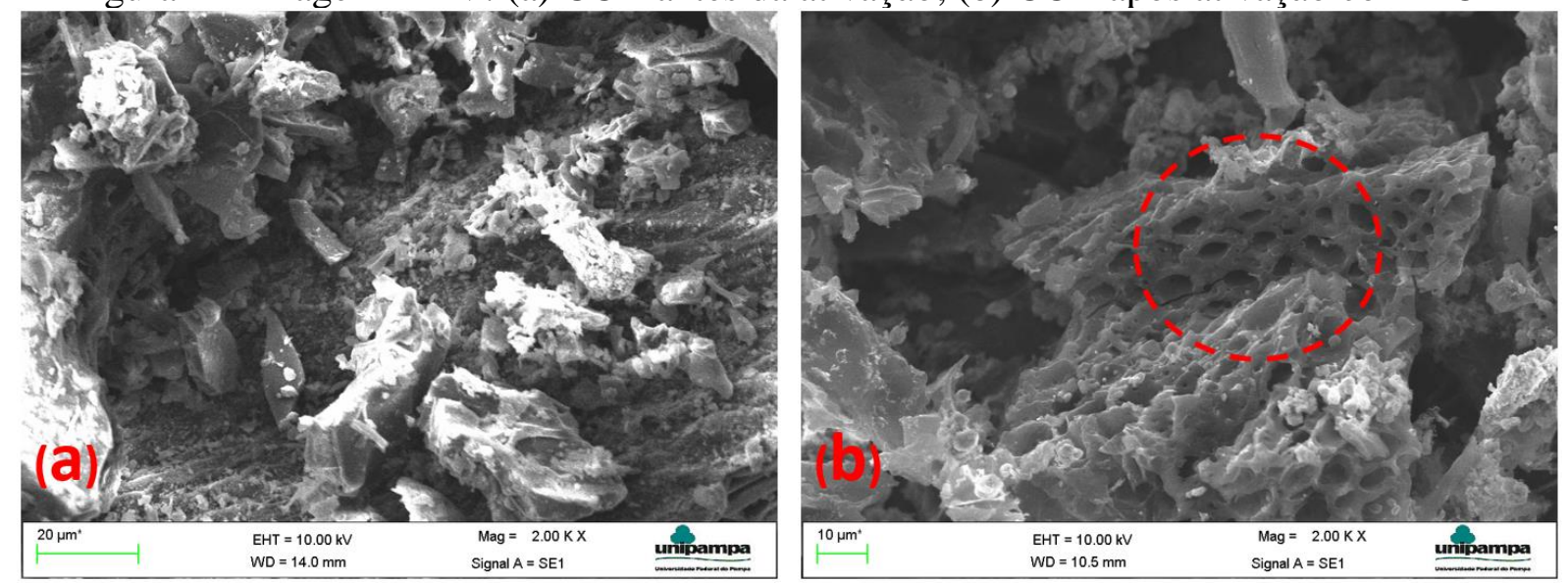

\subsection{Espectroscopia Raman (RAMAN)}

A técnica de espectroscopia de espalhamento Raman tem sido largamente utilizada na caracterização de materiais carbonosos. Com recursos de micro focalização, as investigações são bastante precisas, identificando as diferentes formas cristalinas e amorfas que podem compor as amostras. A Figura 3 ilustra o espectro Raman da amostra FC 500-60 antes (a) e após (b) a ativação química com $\mathrm{KOH}$ a $850^{\circ} \mathrm{C}$ por 120 minutos (GFN-850). Nota-se que os 


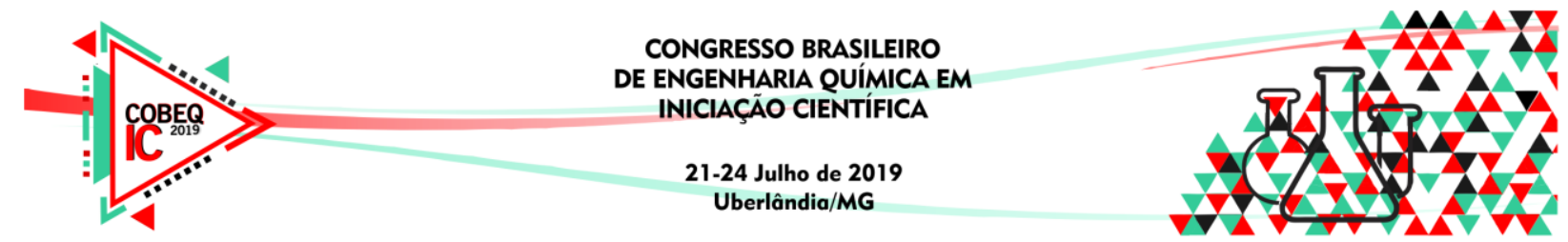

picos característicos, de materiais carbonosos, correspondentes às bandas $G\left(1580 \mathrm{~cm}^{-1}\right) \mathrm{e}$ banda $\mathrm{D}\left(1350 \mathrm{~cm}^{-1}\right)$ estão mais resolvidos após a ativação com $\mathrm{KOH}$, o qual pode ser atribuído à presença de poros, tal como mostrado na Figura 2. Por outro lado, a banda 2D localizada em $\sim 2690 \mathrm{~cm}^{-1}$, característica do grafeno, está presente após a ativação química com KOH. Estes resultados estão de acordo com resultados reportados na literatura de Muramatsu et al (2014) e Sankar et al (2017).

Figura 3 - Espectro Raman da cinza antes (a) e após (b) ativação com KOH

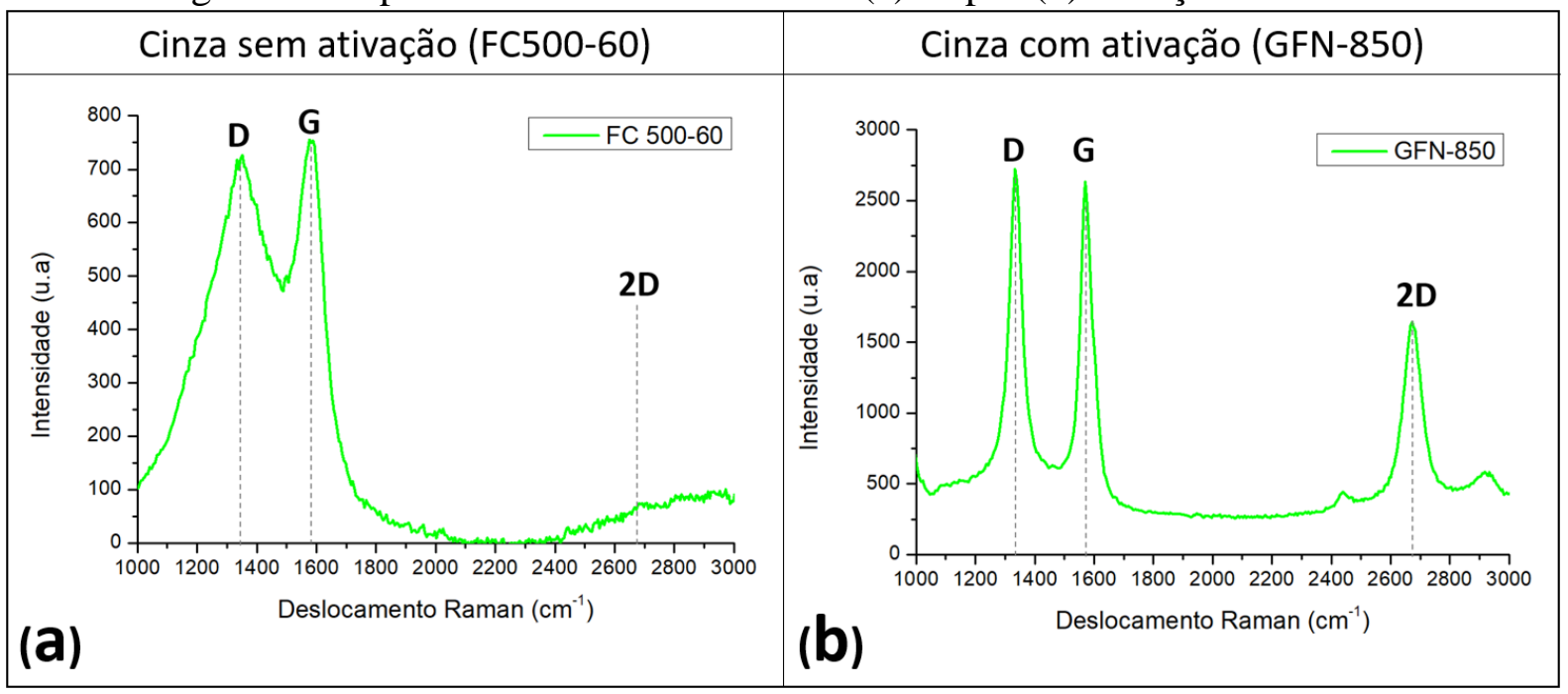

\section{CONCLUSÃO}

Diante de todas as análises realizadas, experimentais e teóricas, é possível concluir que a melhor temperatura de queima para obtenção de uma CCA com alto teor de carbono é 500 ${ }^{\circ} \mathrm{C}$ e, visto que todos os métodos de queima no estudo apresentaram cinza com maior teor de carbono nessa faixa de temperatura. O grafeno obtido através da cinza da CCA apresentou picos definidos das bandas $G, D$ e 2D correspondentes do nano material.

\section{REFERÊNCIAS}

MURAMATSU, H., KIM, Y.A., YANG, K.S., CRUZ-SILVA, R., TODA, I., YAMADA, T., TERRONES, M., ENDO, M., HAYASHI, T. AND SAITOH, H. Rice Husk-Derived Graphene with Nano-Sized Domains and Clean Edges. Small. 2014.

SANKAR, S.; LEE, H.; JUNG, H.; KIM, A.; AHMED, A.T.A.; INAMDAR, A.I.; KIM, H.; LEE, S.; IM, H.; KIM, D.Y. Ultrathin graphene nanosheets derived from rice husks for sustainable supercapacitor electrodes. New Journal of Chemistry, 41(22). 2017.

WANG, L.L., WANG, X., ZOU, B., MA, X., QU, Y., RONG, C., LI, Y., SU, Y., WANG, Z. Preparation of carbon black from rice husk by hydrolysis, carbonization and pyrolysis. Bioresour. Technol. 102. 2011.Lillo-Ródenas et al. (2002)

AZIZI, S.N; YOUSEFPOUR, M. Shynthesis of zeolites NaA and analcime using rice husk ash as sílica source without using organic tamplate. J Mater Sci, 45. 2010. 